\title{
Enriquecimento de floresta em restauração por meio de semeadura direta de lianas ${ }^{1}$
}

\author{
Jeanne Marie Garcia Le Bourlegat ${ }^{2,5}$, Sergius Gandolfi², Pedro Henrique Santim Brancalion ${ }^{3}$, \\ Carlos Tadeu dos Santos Dias ${ }^{4}$
}

Recebido: 23.05.2013; aceito: 15.08.2013

\begin{abstract}
Diversity enhancement of a forest under restoration through direct seeding of lianas). The aim of this study was to analyze the feasibility of direct seeding of liana species in the understory of a restoring forest. Seeds of the species Phanera sp., Macfadyena unguis-cati (L.) A.H. Gentry, and Peltastes peltatus (Vell.) Woodson were submitted to germination tests considering the presence and absence of light and temperature cycle variation. These species were also planted under a canopy of four tree species in a 21-year-old restored forest in Iracemápolis, São Paulo State, Brazil. Macfadyena unguis-cati was the only species presenting a positive photoblastic characteristic, while the two other tested species were not affected by light or temperature cycle. In the understory, Peltastes peltatus did not emerge. In the two other species, seed germination was not influenced by the selected tree species. These results suggest that despite the low rate of emergence, it is possible to enhance the diversity of forest by restoring it through direct seeding of some liana species.
\end{abstract}

Key words: forest restoration, germination, seasonal semideciduous forests, understorey

RESUMO - (Enriquecimento de floresta em restauração por meio de semeadura direta de lianas). Este trabalho objetivou verificar a viabilidade da semeadura direta de lianas no sub-bosque de floresta plantada em restauração. Para isso, sementes das espécies Phanera sp., Macfadyena unguis-cati (L.) A.H. Gentry and Peltastes peltatus (Vell.) Woodson, de lianas foram submetidas a testes de germinação com e sem luz, e com e sem alternância de temperatura. Posteriormente, essas espécies foram semeadas sob a copa de quatro espécies arbóreas em uma Floresta Estacional Semidecidual em restauração no Município de Iracemápolis, SP, Brasil. Em laboratório, apenas Macfadyena unguis-cati apresentou característica de fotoblastismo positivo. As outras espécies não tiveram germinação limitada pela ausência de luz e alternância de temperatura. No sub-bosque, Peltastes peltatus não emergiu; as outras duas espécies não tiveram a emergência alterada em função das espécies arbóreas do dossel sob as quais foram semeadas. Esses resultados sugerem que, apesar da baixa taxa de emergência, é possível o enriquecimento de florestas em processo de restauração a partir da semeadura direta de algumas espécies de lianas.

Palavras-chave: floresta estacional semidecídua, germinação, restauração florestal, sub-bosque

\section{Introdução}

Vários projetos de restauração ecológica vêm sendo desenvolvidos nas últimas décadas com significativos avanços técnicos e científicos (Lamb et al. 2005, Rodrigues et al. 2009, Calmon et al. 2011, Holl \& Aide 2011). Contudo, os monitoramentos de áreas restauradas no Estado de São Paulo têm demonstrado pouca eficácia devido à baixa riqueza e diversidade encontrada no estrato regenerante (Souza 2000, Siqueira 2002, Sorreano 2002, Barbosa et al. 2007).

O elevado grau de fragmentação do Bioma Mata Atlântica (Ribeiro et al. 2009) e o isolamento das áreas em processo de restauração, geralmente imersas em matriz agrícola pouco permeável ao fluxo de fauna

1. Parte da Dissertação de Mestrado da primeira Autora

2. Universidade de São Paulo, Escola Superior de Agricultura "Luiz de Queiroz", Laboratório de Ecologia e Restauração Florestal, Caixa Postal 9, 13418-900 Piracicaba, SP, Brasil

3. Universidade de São Paulo, Escola Superior de Agricultura "Luiz de Queiroz", Departamento de Ciências Florestais, Caixa Postal 9, 13418-900 Piracicaba, SP, Brasil

4. Universidade de São Paulo, Escola Superior de Agricultura "Luiz de Queiroz", Departamento de Ciências Exatas, Caixa Postal 9, 13418-900 Piracicaba, SP, Brasil

5. Autor para correspondência: jeanne_glb@yahoo.com.br 
(Siqueira 2002), podem explicar parte do insucesso verificado.

Nesse contexto, torna-se importante o enriquecimento dessas áreas com outras espécies e formas de vida com o fim de garantir o estabelecimento de diversidade vegetal e funcional, e a autossustentabilidade do ecossistema criado. Por isso, tem se apresentado como próximo desafio da restauração florestal a inserção de outras formas de vida em seus projetos (Belotto et al. 2009, Rodrigues et al. 2009), cujos estudos preliminares já têm mostrado resultados promissores, como a regeneração de lianas provenientes de banco de sementes alóctone (Nave 2005, Jakovac 2007), a sobrevivência de $100 \%$ das mudas de lianas (Santos 2011) e de até 96,7\% de epífitas adultas (Duarte 2013), a partir de transplante para enriquecimento de áreas em processo de restauração florestal.

Dentre essas outras formas de vida, destacam-se as lianas, trepadeiras lenhosas que iniciam seu desenvolvimento como plântulas terrestres, mas que dependem das árvores como suporte para crescer em altura e alcançar o dossel (Schnitzer \& Bongers 2002, Gerwing 2004). Elas constituem um importante componente da estrutura da floresta tropical, com mais de $25 \%$ da diversidade de espécies lenhosas (Gentry 1991, Addo-Fourdjour 2009). Particularmente na Floresta Estacional Semidecidual, onde já foram encontradas até 148 espécies de trepadeiras (Udulutsch et al. 2004), podendo corresponder a 52,5\% da riqueza de espécies lenhosas (Santos et al. 2009).

As lianas apresentam um papel importante na dinâmica florestal, contribuindo nos processos de transpiração e sequestro de carbono (Schintzer \& Bongers 2002), na ciclagem de nutrientes (Putz 1983, 1984, Penalosa 1984, Hegarty 1991) e na estabilidade do microclima florestal na estação fria e seca, melhorando as condições de germinação e estabelecimento de plântulas de espécies arbóreas, além de exercer um efeito protetor frente aos predadores de sementes (Savage 1992, Vieira \& Scariot 2006, Jordão 2009). Além disso, parte das espécies de lianas apresentam picos de floração e frutificação alternados aos das espécies arbóreas na Floresta Estacional Semidecídua, garantindo a oferta de recursos alimentares à fauna de polinizadores e dispersores em períodos de escassez (Galetti \& Pedroni 1994, Galetti et al.1994, Morellato \& LeitãoFilho 1996) e também contribuem na formação da complexidade estrutural da floresta, servindo para a movimentação, abrigo e coexistência da fauna arborícola não alada, ao criarem verdadeiras estradas suspensas entre as copas das árvores (Giné 2009, Putz 2009, Melo et al. 2011).

Contudo, a utilização de espécies de lianas na restauração ecológica ainda é incipiente e requer o desenvolvimento de técnicas adequadas à sua inserção e de conhecimentos sobre a ecofisiologia das espécies, que ainda são escassos.

As estratégias de enriquecimento utilizadas atualmente são as de transferência de serrapilheira, banco de sementes alóctones e de plântulas, plantio de mudas ou semeadura direta. Entretanto, ainda não se sabe qual dessas técnicas seria a mais adequada para esse grupo vegetal. A semeadura direta, técnica ainda em desenvolvimento mesmo para espécies arbóreas, pode reduzir os custos da implantação do projeto de restauração (Engel \& Parrotta 2001, Lamb et al. 2005, Isernhagen 2010, Aguirre 2012), evitando a fase de produção da muda no viveiro e o seu transporte.

Diante disto, o objetivo deste trabalho foi verificar a viabilidade da semeadura direta das lianas no sub-bosque de uma floresta, plantada em 1987, em processo de restauração ecológica.

\section{Material e métodos}

Frutos das lianas Macfadyena unguis-cati (L.) A.H. Gentry, Peltastes peltatus (Vell.) Woodson e Phanera sp., foram coletados em diferentes fragmentos de Floresta Estacional Semidecídua nas proximidades de Piracicaba (SP) durante os meses de fevereiro a julho de 2008. Estas espécies foram selecionadas por não apresentarem caráter agressivo nas áreas em que foram naturalmente observadas e floração durante o primeiro semestre do ano, alternada à da comunidade arbórea.

As identificações taxonômicas foram realizadas com o auxílio de especialistas do Herbário Rioclarense (HRCB).

Os frutos coletados foram mantidos em estufa com circulação e renovação de ar (MARCONI MA 035) a $35^{\circ} \mathrm{C}$ por três dias para possibilitar a abertura forçada dos mesmos e a consequente extração das sementes. Foram descartadas as sementes que apresentavam algum sinal de predação ou má formação do embrião, verificada visualmente através da morfologia externa. As demais foram armazenadas em saco de papel no interior de ambiente controlado $\left(20{ }^{\circ} \mathrm{C}\right.$ e $40 \%$ de umidade relativa do ar).

Foram desenvolvidos dois experimentos com as sementes de lianas, de setembro a dezembro 
de 2008, no Laboratório de Análise de Sementes do Departamento de Produção Vegetal, da Escola Superior de Agricultura "Luiz de Queiroz", USP; e outro em uma floresta plantada em processo de restauração.

Experimento 1 - Sementes das três espécies de lianas foram submetidas a quatro tratamentos a fim de verificar se a ausência de luz e a alternância de temperatura interferiam na sua capacidade de germinação: presença de luz e temperatura constante; ausência de luz e temperatura constante; presença de luz e temperatura alternada e ausência de luz e temperatura alternada.

A semeadura foi realizada em caixas de plástico transparente $(11 \times 11 \times 3 \mathrm{~cm})$ sobre duas folhas de papel de filtro umedecidas com água até atingir $60 \%$ de sua capacidade de retenção, de acordo com Brasil (2009).

Para os dois primeiros tratamentos, as caixas foram mantidas em germinador de sementes (MARCONI 348 EB) sob temperatura constante de $25{ }^{\circ} \mathrm{C}$, na presença de luz (caixas plásticas transparentes) e na ausência de luz (caixas plásticas pretas). Para os demais tratamentos, as caixas foram mantidas em câmara de germinação (MARCONI MA 402) na presença de luz com temperatura alternando a cada 12 horas entre 20 e $35^{\circ} \mathrm{C}$. Para o tratamento sem luz, foram utilizadas caixas plásticas pretas.

Foram utilizadas quatro caixas (repetições) 25 sementes para cada tratamento, 100 sementes por espécie por tratamento, totalizando 400 sementes de cada espécie.

As sementes foram umedecidas com água sempre que o substrato se apresentava seco. Para as caixas pretas esse procedimento foi feito em sala escura, sob luz verde. A avaliação da germinação foi realizada a cada dois dias até sua estabilização, seguindo os critérios indicados nas Regras para Análise de Sementes (Brasil 2009); com os dados obtidos, foi calculada a porcentagem de germinação.

Experimento 2 - Foi realizada a semeadura direta das três espécies de lianas no sub-bosque de uma floresta em processo de restauração, plantada em 1987. Aárea faz parte de um projeto de recuperação do entorno da represa de abastecimento público do Município de Iracemápolis, SP, onde foram introduzidas 140 espécies, entre nativas e algumas exóticas, em uma faixa marginal ao reservatório com 50 metros de largura. Seu entorno é dominado pelo cultivo de cana-de-açúcar, do qual é separada por uma estrada que funciona como aceiro (Rodrigues et al. 1992).

A seleção da área levou em conta a idade do plantio, que à época deste trabalho tinha 21 anos, e a fisionomia florestal do local, constituído por dossel bem definido variando entre 7 a 17 metros de altura, com presença de indivíduos emergentes, clareiras e sub-bosque (Castanho 2009).

O clima regional é do tipo Cwa, clima quente, com temperatura média do mês mais quente do verão acima de $22{ }^{\circ} \mathrm{C}$ e com inverno seco, sendo o total de chuvas do mês mais seco inferior a $30 \mathrm{~mm}$ e temperatura média do mês mais frio inferior a $18^{\circ} \mathrm{C}$, segundo a classificação de Köppen (Setzer 1966), com precipitação variando de 1.100 a $1.700 \mathrm{~mm}$ por ano. Os solos presentes na região são classificados como Latossolo Vermelho distrófico e Latossolo Vermelho eutrófico (Projeto Radam Brasil 1983, Rodrigues et al. 1992, Prado 2003).

Dentro da área de estudo, foram escolhidas quatro espécies arbóreas de dossel, Ceiba speciosa (A.St.-Hil.) Ravenna, Schizolobium parahyba (Vell.) S.F. Blake, Piptadenia gonoacantha (Mart.) J.F. Macbr. e Triplaris americana L., que possuem diferentes arquiteturas de copa e padrões de perenidade ou deciduidade que potencialmente poderia criar distintos micro-sítios de regeneração espécie-específicos sob a projeção de suas copas. Dessa maneira, essas espécies de dossel poderiam, potencialmente, interferir de forma diferencial na capacidade de germinação e sobrevivência das plantas que se desenvolvem sob elas, agindo como filtro em relação a essas espécies. Nesse sentido, se as espécies de lianas apresentassem a preferência ou limitação da emergência de plântulas sob determinadas espécies arbóreas do dossel ficaria constatada a necessidade de uma pré-seleção de locais no sub-bosque para a semeadura de espécies de enriquecimento em florestas plantadas e, em caso contrário, dispensa de uma pré-seleção.

Foram marcados 12 indivíduos de cada espécie arbórea selecionada, sob os quais foram semeadas as três espécies de lianas, totalizando 48 árvores (três espécies de lianas $\times$ quatro espécies arbóreas $\times$ quatro repetições).

Sob a projeção da copa de cada um dos 48 indivíduos marcados foi feita, com enxada, uma faixa de limpeza no solo na forma de circunferência, centralizada no tronco da árvore, de raio igual a $64 \mathrm{~cm}$, onde foram realizados os experimentos.

Foram distribuídas 25 sementes de uma espécie de liana na faixa, com espaçamento de $16 \mathrm{~cm}$ entre 
elas, totalizando 400 sementes de cada espécie $(25$ sementes $\times$ quatro espécies arbóreas $\times$ quatro repetições).

As sementes foram colocadas a uma profundidade de $0,5 \mathrm{~cm}$ da superfície do solo e sobre cada uma foi colocada uma fina camada de terra da própria faixa, a fim de não deixar a semente totalmente exposta. Para demarcar o local da semeadura, ao lado de cada semente foi colocado um palito de madeira de $10 \mathrm{~cm}$ de comprimento.

O monitoramento da semeadura direta foi realizado mensalmente avaliando-se do número de plântulas (emergência) de cada espécie de liana presente sob as diferentes espécies arbóreas do dossel por um período de 210 dias (dezembro de 2008 a junho de 2009).

Análise dos dados - Os valores de emergência obtidos na semeadura direta não atenderam aos requisitos de normalidade e homogeneidade de variância, por isto foram transformados por potência ótima de Box-Cox.

Os resultados de germinação e de emergência de cada experimento foram submetidos à Análise de Variância (ANOVA), sendo que as médias foram comparadas pelo teste Tukey a 5\% de significância (Zar 1984).

\section{Resultados e Discussão}

De modo geral, as lianas são encontradas em abundância em clareiras e bordas de mata, ambientes esses com maior irradiância e alternância de temperatura quando comparados com o sub-bosque, o que leva à ideia de que esse grupo vegetal é dependente desses fatores para a germinação (Hegarty \& Caballé 1991). Entretanto, os resultados obtidos no presente estudo revelaram que cada espécie de liana responde diferentemente às condições ambientais a que estão submetidas durante a germinação, indicando que esse grupo vegetal não apresenta homogeneidade quanto à necessidade de luz e alternância de temperatura.

Em estufa, as sementes de Phanera sp. não apresentaram diferença significativa de germinação entre os tratamentos (tabela 1). A capacidade de germinar mesmo em condição de ausência de luz e sem a necessidade de flutuações de temperatura indica que a espécie não teria sua germinação limitada por esses fatores no interior da floresta, onde a radiação solar é alterada durante a passagem pelo dossel, com a quantidade e a qualidade da radiação fotossinteticamente ativa afetada (Turnbull \& Yates 1993, Chazdon et al. 1996). No entanto, não se pode descartar, devido a germinação de $19 \%$ no escuro, que a espécie possa também germinar, em pequena proporção, no sub-bosque.

Para Macfadyena unguis-cati, a ausência de alternância de temperatura também não foi fator limitante à germinação (tabela 1). Entretanto, suas sementes não germinaram na ausência de luz em temperatura constante. No escuro, com alternância de temperatura, houve $19 \%$ de germinação; entretanto, este resultado foi significativamente menor que o encontrado nos dois tratamentos com luz, o que revela que as sementes dessa espécie são fotoblásticas positivas.

A aptidão dessa espécie em detectar mudanças no microclima, correspondentes às condições favoráveis para germinação e estabelecimento, pode capacitá-la a ter um controle de onde e quando haverá a germinação, prevenindo que ela germine em local e período desfavorável para o estabelecimento da plântula (Fenner \& Thompson 2005), podendo ficar limitada à clareira ou à borda florestal.

As sementes de Peltastes peltatus, por sua vez, não tiveram a germinação alterada pelas condições de luz em laboratório (tabela 1), indicando que o sombreamento do interior florestal não é limitante à

Tabela 1. Germinação média das espécies de lianas estudadas sob diferentes condições de luz e temperatura, Iracemópolis, SP, Brasil. Médias seguidas de *, na horizontal, indicam que há diferença significativa, pelo teste de Tukey, ao nível de significância de $0,05^{* *}$, ns indica que a diferença não é significativa.

Table 1. Average germination of liana species under different conditions of light and temperature, Iracemópolis, São Paulo State, Brazil. Means followed by *, horizontally, indicate significant difference by Tukey test at a significance level of $0.05^{* *}$, ns indicates that the difference is not significant.

\begin{tabular}{|c|c|c|c|c|c|}
\hline Espécie & $\begin{array}{c}\text { Com luz/ temper } \\
\text { constante }\end{array}$ & $\begin{array}{l}\text { luz/ tempe } \\
\text { alternada }\end{array}$ & $\begin{array}{l}\text { luz/ temper } \\
\text { constante }\end{array}$ & $\begin{array}{l}\text { luz/ temperatura } \\
\text { alternada }\end{array}$ & Média \\
\hline Phanera sp. & 0,60 & 0,64 & 0,66 & 0,68 & 0,65 \\
\hline Macfadyena unguis-cati & 0,81 & 0,81 & $0,00 *$ & $0,19 *$ & 0,45 \\
\hline Peltastes peltatus & 0,83 & $0,01 *$ & 0,59 & $0,15^{\mathrm{ns}}$ & 0,40 \\
\hline
\end{tabular}


germinação. Porém, tiveram redução significativa da germinação na condição de alternância de temperatura, o que pode ser explicado pela existência de uma faixa de temperatura ótima para germinação, específica para cada espécie (Fenner 2000). Nesse caso, a temperatura mínima para germinação de $P$. peltatus deve estar acima de $20^{\circ} \mathrm{C}$ e, portanto, quando submetida à temperatura de $25^{\circ} \mathrm{C}$ conseguiu atingir a germinação, mas quando no tratamento de temperatura alternada, em que permanecia por muitas horas abaixo da temperatura requerida, não era capaz de germinar. Todavia, considerando que, de acordo com Whitmore (1990), a temperatura do solo no sub-bosque sob dossel fechado em Florestas Tropicais permanece por volta de $25^{\circ} \mathrm{C}$, as sementes de P. peltatus também não teriam a germinação limitada pela temperatura naquele ambiente.

Com base nos resultados obtidos, a ausência de luz e de alternância de temperatura do interior florestal não são fatores limitantes para a germinação de duas das três espécies estudadas.

No experimento de semeadura direta, não foi verificada diferença significativa do total de plântulas de lianas entre os sub-bosques das quatro espécies arbóreas do dossel $\left(F_{(3,11)}=1,16 ; p=0,3387\right)$, bem como na interação entre cada espécie de liana e os tratamentos $\left(F_{(3,11)}=2,01 ; p=0,0897\right)$, indicando que não haveria necessidade de uma seleção prévia do local para a semeadura das espécies de trepadeiras selecionadas em relação às espécies do dossel. Experimentos semelhantes realizados com mais espécies de lianas e árvores do dossel poderão reforçar se essa é uma tendência geral.

Phanera sp. apresentou porcentagem média de emergência de plântulas de 6\% (tabela 2), consideravelmente inferior à porcentagem de germinação observada em laboratório para o mesmo

Tabela 2. Média e desvio padrão da emergência de plântulas das três espécies de lianas estudadas sob diferentes espécies arbóreas na área de estudo em Iracemápolis, SP, Brasil.

Table 2. Means and standard deviation of seedling emergence of three liana species under different tree species in the studied area in Iracemápolis, São Paulo State, Brazil.

\begin{tabular}{lcc}
\hline Espécies & $\begin{array}{c}\text { Emergência } \\
\text { média }\end{array}$ & Desvio padrão \\
\hline Phanera sp. & 0,06 & $\pm 0,04$ \\
Macfadyena unguis-cati & 0,39 & $\pm 0,12$ \\
Peltastes peltatus & 0,00 & 0,00 \\
\hline
\end{tabular}

lote de sementes $(64,5 \%)$ (tabela 1$)$. Isto pode ser explicado pela variedade de condições ambientais presentes no campo, que não se restringem apenas à presença de luz e temperatura, como déficit de umidade, por exemplo. Outro ponto importante a destacar é que o monitoramento da semeadura direta foi feito pela observação da emergência das plântulas, por isso, a não emergência também pode ser resultado da destruição das sementes por predadores. Isso é corroborado pelo fato de que não foram encontradas sementes no solo além daquelas que emergiram, sugerindo forte influência de predação, assim como verificado por Sanches (1997), em que a perda da viabilidade de algumas espécies chegou a 100\%. Para Dalling (2003), a presença de herbívoros e patógenos é crucial para afetar o recrutamento ou não das espécies nas florestas tropicais.

Isto também pode explicar o resultado obtido para P. peltatus, que não apresentou nenhuma plântula no experimento de semeadura direta no sub-bosque florestal, e nenhuma semente não germinada foi encontrada ao fim do experimento.

A espécie $M$. unguis-cati foi a que apresentou maior porcentagem de emergência média de plântulas $(39,25 \%)$ entre as estudadas (tabela 2), com máximas e mínimas de $16 \%$ e $60 \%$ (tabela 3 ), diferente do esperado, tendo em vista que em laboratório a espécie apresentou caráter fotoblástico positivo. Este resultado pode estar associado à ocorrência de "sunflecks" (fachos de luz solar direta, de curta duração e pequena dimensão, que se deslocam pelo sub-bosque) e interferem significativamente na germinação de sementes por seu grande conteúdo energético e alta razão V/VE (Turnbull \& Yates 1993, Vazquez-Yanes \& Orozco-Segovia 1993); e à estrutura do dossel onde foram semeadas, por se tratar de uma faixa de floresta plantada com apenas 50 metros de largura, com menor densidade de plantas no sub-bosque e a presença de várias espécies arbóreas pioneiras mortas que abrem clareiras no dossel e permitem a maior entrada de luz. Pode ainda ser uma expressão do potencial, observado em laboratório, que essa espécie tem de apresentar uma baixa taxa de germinação no escuro em temperaturas alternadas (19\%), o que a permitiria também germinar no sub-bosque, mesmo sob sombra densa.

Estes resultados revelaram que a semeadura direta de lianas no interior florestal não está aparentemente restrita à utilização de espécies tolerantes ao sombreamento como se esperava, mas que devido 
Tabela 3. Distribuição do número total de plântulas de cada espécie de liana sob cada espécie arbórea do dossel, Iracemápolis, SP, Brasil. Table 3. Distribution of the total number of seedlings for each species of liana under each arboreal species of the canopy, Iracemápolis, São Paulo State, Brazil.

\begin{tabular}{lccc}
\hline Árvores & Phanera sp. & Macfadyena unguis-cati & Peltastes peltatus \\
\hline Ceiba speciosa & 9 & 38 & 0 \\
Piptadenia gonoacantha & 3 & 43 & 0 \\
Schizolobium parahyba & 9 & 29 & 0 \\
Triplaris americana & 5 & 46 & 0 \\
\hline
\end{tabular}

às condições ambientais particulares das áreas em processo de restauração, é possível a emergência de plântulas de espécies fotoblásticas positivas, aumentando o leque de espécies a ser utilizado nessa estratégia.

Embora tenha sido obtida baixa taxa de emergência neste experimento, assim como verificado por Isernhagen (2010) na semeadura direta de espécies arbóreas de enriquecimento, cujas taxas médias variaram de $5 \%$ a $6 \%$, ficou demonstrado que é possível realizar o enriquecimento de florestas plantadas em processo de restauração a partir da semeadura direta para algumas espécies de lianas; o esforço é relativamente baixo quando comparado à produção de mudas em viveiro, e há ganho efetivo em diversidade, uma vez que o objetivo é a introdução de espécies antes inexistentes na área, ainda que com pequeno número de indivíduos.

No entanto, por ser uma técnica ainda em desenvolvimento, mais experimentos devem ser feitos, por exemplo, com maior número de espécies de lianas, diferentes densidades de sementes, uso de protetores físicos, pré-tratamentos das sementes e semeadura em diferentes períodos do ano.

Por fim, tendo em vista a importância desse grupo vegetal, especialmente na Floresta Estacional Semidecidual, é essencial o prosseguimento de estudos que fundamentem a inserção de espécies de lianas na restauração ecológica com o devido cuidado de não utilizar espécies potencialmente agressivas, que poderiam levar a resultados opostos ao desejado, como a redução da diversidade de espécies não pioneiras, conforme apontado por Schnitzer \& Carson (2010).

\section{Agradecimentos}

Ao CNPq, pela Bolsa de Mestrado concedida à primeira Autora e à Cristina Yuri Vidal, pelas sugestões.

\section{Literatura citada}

Addo-Fordjour, P. 2009. Liana species richness, abundance and relationship with trees in the Bobiri forest reserve, Ghana: Impact of management systems. Forest Ecology and Management 257: 1822-1828.

Aguirre, A.G. 2012. Avaliação do potencial de regeneração natural e o uso de semeadura direta e estaquia com técnicas de restauração. Dissertação de Mestrado, Universidade de São Paulo, Piracicaba.

Barbosa, L.M., Barbosa, K.C., Barbosa, J.M., Fidalgo, A.O., Rondon, J.N., Neves Junior, N., Martins, S.E., Duarte, R.R., Casagrande, J.C. \& Carlone, N.P. 2007. Estabelecimento de políticas públicas para recuperação de áreas degradadas no Estado de São Paulo: o papel das instituições de pesquisa e ensino. Revista Brasileira de Biociências 5: 162-164.

Bellotto, A., Viani, R.A.G., Gandolfi, S. \& Rodrigues, R.R. 2009. Inserção de outras formas de vida no processo de restauração. In: R.R. Rodrigues, P.H.S. Brancalion \& I. Isernhagen (eds.). Pacto pela restauração da Mata Atlântica: referencial dos conceitos e ações de restauração florestal. Instituto BioAtlântica, São Paulo, pp. 55-61.

Brasil. 2009. Ministério da Agricultura, Pecuária e Abastecimento. Secretaria de Defesa Agropecuária. Regras para análise de sementes. Mapa/ACS, Brasília.

Calmon, M., Brancalion, P.H. S., Paese, A., Aronson, J., Castro, P., Silva, S.C. \& Rodrigues, R.R. 2011. Emerging threats and opportunities for large-scale ecological restoration in the atlantic forest of Brazil. Restoration Ecology 19: 154-158.

Castanho, G.G. 2009. Avaliação de dois trechos de uma Floresta Estacional Semidecidual restaurada por meio de plantio, com 18 e 20 anos, no Sudeste do Brasil. Dissertação de Mestrado, Universidade de São Paulo, Piracicaba.

Chazdon, R.L., Pearcy, R.W., Lee, D.W. \& Fetcher, N. 1996. Photosynthetic responses of Tropical Forest plants to contrasting light environments. In: S.S. Mulkey, R.L. Chazdon \& A.P. Smith (eds.). Tropical Forest Plant Ecophysiology. Chapman and Hall, New York, pp. 5- 55.

Dalling, J.W. 2003. Ecologia de semillas. In: M.R. Guariguata \& G.H. Kattan (eds.). Ecología y conservación de bosques neotropicales. Ediciones LUR, Cartago, pp. 345-375. 
Duarte, M.M. 2013. Transplante de epífitas entre Florestas Estacionais Semideciduais para enriquecimento de floresta restaurada. Dissertação de Mestrado, Universidade de São Paulo, Piracicaba.

Engel, V.L. \& Parrotta, J.A. 2001. An evaluation of direct seeding for reforestation of degraded lands in central Sao Paulo State, Brazil. Forest Ecology and Management 152: 169-181.

Fenner, M. \& Thompson, K. 2005. The Ecology of Seeds. Cambridge University Press, Cambridge.

Fenner, M. 2000. Seeds: the ecology of regeneration in plant communities. CABI Publishing, New York.

Galetti, M. \& Pedroni, F. 1994. Seasonal diet of capuchin monkeys (Cebus apella) in a Semideciduous Forest in southeast Brazil. Journal of Tropical Ecology 10: 27-39.

Galetti, M., Pedroni, F. \& Morellato, L.P.C. 1994. Diet of the brown howler monkey Alouatta fusca in a forest fragment in southeastern Brazil. Mammalia 48: 1-18.

Gentry, A.H. 1991. The distribution and evolution of climbing plants. In: F.E. Putz \& H.A. Mooney. The biology of vines. Cambridge University Press, Cambridge. pp. 3-52.

Gerwing, J.J. 2004. Life history diversity among six species of canopy lianas in an old-growth forest of the eastern Brazilian Amazon. Forest Ecology and Management 190: 57-72.

Giné, G.A.F.E. 2009. Ecologia e comportamento do ouriço-preto (Chaetomys subspinosus, Olfers 1818) em fragmentos de Mata Atlântica do município de Ilhéus, sul da Bahia. Tese de Doutorado, Universidade de São Paulo, Piracicaba.

Hegarty, E.E. 1991. Leaf litter production by lianes and trees in a Sub-Tropical Australian Rain Forest. Journal of Tropical Ecology 7: 201-214.

Hegarty, E.E. \& Caballé, G. 1991. Distribution and abundance of vines in forest communities. In: Putz, F.E. \& Mooney, H.A. The Biology of Vines. Cambridge University Press, Cambridge, pp. 313-336.

Holl, K.D. \& Aide, T.M. 2011. When and where to actively restore ecosystems? Forest Ecology and Management 261: $1558-1563$.

Isernhagen, I. 2010. Uso de semeadura direta de espécies arbóreas nativas para restauração florestal de áreas agrícolas, sudeste do Brasil. Tese de Doutorado, Universidade de São Paulo, Piracicaba.

Jakovac, A.C.C. 2007. O uso do banco de sementes florestal contido no topsoil como estratégia de recuperação de áreas degradadas. Dissertação de Mestrado, Universidade Estadual de Campinas, Campinas.

Jordão, S.M.S. 2009. Manejo de lianas em bordas de floresta estacional semidecidual e de cerradão, Santa Rita do Passa Quatro, SP. Tese de Doutorado, Universidade de São Paulo, Piracicaba.

Lamb, D., Erskine, P.D. \& Parrotta, J.A. 2005. Restoration of degraded tropical forest landscapes. Science 310: 1628-32.
Melo, G.L., Sponchiado, J., Machado, A.F. \& Cáceres, N.C. 2011. Small-mammal community structure in a South American deciduous Atlantic Forest. Community Ecology 12: 58-66.

Morellato, P.C. \& Leitão Filho, H.F. 1996. Reproductive phenology of climbers in a Southeastern Brazilian Forest. Biotropica 28: 180-191.

Nave, A.G. 2005. Banco de sementes autóctone e alóctone, resgate de plantas e plantio de vegetação nativa na Fazenda Intermontes, município de Ribeirão Grande. Tese de Doutorado, Universidade de São Paulo, Piracicaba.

Penalosa, J. 1984. Basal branching and vegetative spread in two tropical rain forest lianas. Biotropica 16: 1-9.

Prado, H. 2003. Solos do Brasil: gênese, morfologia, classificação, levantamento agrícola e geotécnico. 3 ed. FUNEP, Piracicaba.

Projeto RadamBrasil. 1983. Folhas SF. 23/24: Rio de Janeiro/Vitória. DNPM, Rio de Janeiro.

Putz, F.E. 1983. Liana biomass and leaf area of a Tierra Firme Forest in the Rio Negro Basin, Venezuela. Biotropica 15: 185-189.

Putz, F.E. 1984. The natural history of lianas on Barro Colorado Island, Panama. Ecology 65: 1713-1724.

Putz, F.E. 2009. Vine Ecology. http//: www.ecology.info/ vines.htm (acesso em 18.04.2013).

Ribeiro, M.C., Metzger, J.P., Martensen, A.C., Ponzoni, F. \& Hirota, M.M. 2009. Brazilian Atlantic forest: how much is left and how is the remaining forest distributed? Implications for conservation. Biological Conservation 142: 1141-1153.

Rodrigues, R.R., Leitão-Filho, H.F. \& Crestana, M.S.M. 1992. Regeneração do entorno da represa de abastecimento de água do Município de Iracemápolis/ SP. In: Anais do Simpósio Nacional de Recuperação de Áreas Degradadas, Curitiba, pp. 406-414.

Rodrigues, R.R., Lima, R.A.F., Gandolfi, S. \& Nave, A. 2009. On the restoration of high diversity forests: 30 years of experience in the Brazilian Atlantic Forest. Biological Conservation 142: 1242-1251.

Sanches, M.C. 1997. Espécies de lianas da Reserva Municipal de Santa Genebra, Campinas, SP: longevidade das sementes e crescimento inicial. Dissertação de Mestrado, Universidade Estadual de Campinas, Campinas.

Santos, K., Kinoshita, L.S. \& Rezende, A.S. 2009. Species composition of climbers in seasonal semideciduous forest fragments of Southeastern Brazil. Biota Neotropica 9: 175-188.

Santos, M.B. 2011. Enriquecimento de uma floresta em restauração através da transferência de plântulas da regeneração natural e da introdução de plântulas e mudas. Tese de Doutorado, Universidade de São Paulo, Piracicaba. 
Savage, M. 1992. Germination of forest species under an anthropogenic vine mosaic in western Samoa. Biotropica 24: 460-462.

Schnitzer, S.A. \& Bongers, F. 2002. The ecology of lianas and their role in forests. Trends in Ecology and Evolution 17: 223-230.

Schnitzer, S.A. \& Carson, W.P. 2010. Lianas suppress tree regeneration and diversity in treefall gaps. Ecology Letters 13: 849-857.

Setzer, J. 1966. Atlas climático e ecológico do Estado de São Paulo. Comissão Interestadual da Bacia do ParanáUruguai e Centrais Elétricas do Estado de São Paulo, São Paulo.

Siqueira, L.P. 2002. Monitoramento de áreas restauradas no interior do Estado de São Paulo, Brasil. Dissertação de Mestrado, Universidade de São Paulo, Piracicaba.

Sorreano, M.C.M. 2002. Avaliação de aspectos da dinâmica de florestas restauradas, com diferentes idades. Dissertação de Mestrado, Universidade de São Paulo, Piracicaba.
Souza, F.M. 2000. Estrutura e dinâmica do estrato arbóreo e da regeneração natural em áreas restauradas. Dissertação de Mestrado, Universidade de São Paulo, Piracicaba.

Turnbull, M.T. \& Yates, D. 1993. Seasonal variation in the red/far-red ratio and photon flux density in an Australian sub-tropical rainforest. Agricultural and Forest Meteorology 64: 111-127.

Udulutsch, R.G., Assis, M.A. \& Picchi, D.G. 2004. Florística de trepadeiras numa floresta estacional semidecídua, Rio Claro - Araras, Estado de São Paulo, Brasil. Revista Brasileira de Botânica 27: 125-134.

Vazquez-Yanes, C. \& Orozco-Segovia, A. 1993. Patterns of seed longevity and germination in the Tropical Rainforest. Annual Review of Ecology and Systematics 24: 69-87.

Vieira, D.L.M. \& Scariot, A. 2006. Effects of logging, liana tangles and pasture on seed fate of dry forest tree species in Central Brazil. Forest Ecology and Management 230: 197-205.

Whitmore, T.C. 1990. An Introduction to Tropical Rain Forest. Oxford University Press, Oxford.

Zar, J.H. 1984. Bioestatiscal analysis. Prentice, New Jersey. 